\title{
A low power clock generator with adaptive inter-phase charge balancing for variability compensation in 40-nm CMOS
}

\author{
U. Schulze, M. Broich, O. Weiss, and T. G. Noll \\ Chair of Electrical Engineering and Computer Systems, RWTH Aachen University, Germany
}

\begin{abstract}
Power dissipation besides chip area is still one main optimization issue in high performance CMOS design. Regarding high throughput building blocks for digital signal processing architectures which are optimized down to the physical level a complementary two-phase clocking scheme (CTPC) is often advantageous concerning ATE-efficiency. The clock system dissipates a significant part of overall power up to more than $50 \%$ in some applications.

One efficient power saving strategy for CTPC signal generation is the charge balancing technique. To achieve high efficiency with this approach a careful optimization of timing relations within the control is inevitable.

However, as in modern CMOS processes device variations increase, timing relations between sensitive control signals can be affected seriously. In order to compensate for the influence of global and local variations in this work, an adaptive control system for charge balancing in a CTPC generator is presented. An adjustment for the degree of charge recycling is performed in each clock cycle. In the case of insufficient recycling the delay elements which define duration and timing position of the recycling pulse are corrected by switchable timing units.

In a benchmark with the conventional clock generation system, a power reduction gain of up to $24.7 \%$ could be achieved. This means saving in power of more than $12 \%$ for a complete number-crunching building block.
\end{abstract}

\section{Introduction}

Designing high performance building blocks for digital signal processing systems is facing increased challenges in modern CMOS generations especially concerning leakage and process parameter variation (Pang et al., 2009; Das et al., 2009; Nassif et al., 2007; Jaffari and Anis, 2006). These challenges of ultra deep submicron technologies have a great impact on well established design strategies. Nevertheless

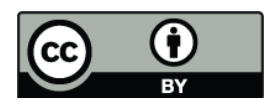

Correspondence to: U. Schulze

(schulze@eecs.rwth-aachen.de) the demand of efficient implementations concerning area, throughput and power dissipation has to be satisfied.

For highly pipelined architectures in many cases a complementary two-phase clocking scheme (CTPC) is the most efficient choice for realizing the synchronization. In such building blocks power dissipation of the logic can often be reduced to $60 \%$, in some applications even down to $50 \%$ of overall power (see Fig. 1, left diagram) This makes the clock system to be the main energy consumer.

In order to reduce clock power an approach for signal generation which performs charge balancing between complementary phases is very promising (Noll and de Man, 1992; Kyriakis-Bitzaros and Nikolaidis, 1997). With this strategy the power efficiency in the clock system can be be enhanced by one third (Fig. 1, right diagram). For a proper functionality of the charge balancing technique, the control signals for buffers and balancing transistors have to be in well adjusted timing relations to each other. Even small shifts between the signals can reduce the amount of recycled charge significantly or cause shorts towards the power supply. As in modern CMOS technologies process variations increase and will in future generations increase even more, power saving clock signal generation will become more complicated.

To overcome the difficulty of timing shifts between sensitive control signals caused by variability we propose in this work an adaptive approach. In a first step, the quality of the charge balancing event is evaluated. If it is not working well timing relations between the control signals are corrected by inserting or skipping little delays resulting in an optimized adjustment of the signals and thus in optimum recycling performance.

In Sect. 2 of this paper the principle of charge balancing is explained. Section 3 describes two main imperfections which can occur within the balancing process. Then in Sect. 4 a new gate for equilibrium detection is presented. Section 5 gives an overview of the complete clock generator implementaion. Finally, in Sect. 6 a benchmarking is made towards two conventional clock systems without parameter adaption. 

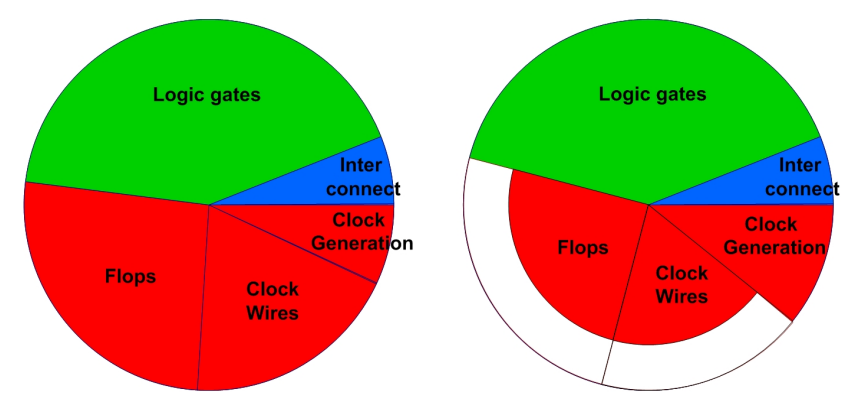

Fig. 1. Power breakdown of FIR-filter with/-out charge balancing.

\section{Inter-phase charge balancing: basic principle}

The mechanism of charge balancing between two complementary phases of one clock signal has been introduced in Noll and de Man (1992). Figure 2 shows the basic circuit including two capacitors which represent the capacitive loads of both complementary signals $\Phi$ and $\bar{\Phi}$. The parasitic elements include capacitances of global and local wiring and the clock inputs of all pipeline elements.

Shorting both phases at the moment of attended switching will equalize the charges on both parasitic capacitors. The voltage equilibrium can thus be reached without taking any current from the supply nodes in a best case. If the hardware overhead for the charge balancing circuits is neglected about $50 \%$ of power saving within the clock network is possible.

In Fig. 3 both complementary phases $\Phi$ and $\bar{\Phi}$ of one clock signal within a CTPC system and the according control signals are illustrated. The balancing mechanism works as follows: to initiate a clock signal transition at first, both tri-state buffers have to be closed making the clock trees floating for a short amount of time $t_{\text {off }}$ (see Fig. 3, control signals $p s$ and ns).

After that, the charge balancing transistor is opened with a balancing pulse of length $t_{\mathrm{cb}}$ (see Fig. 3, control signal $c b_{-} s$ ) until the equilibrium is almost reached.

Finally, with a little, well adjusted overlapping time $t_{\text {ovlp }}$ (see Fig. 3, control signals $n s$ and $p s$ ) two of the four tristate transistors get an impulse to conduct for the rest of the half-cycle. This event will complete the transition.

The optimal choice of $t_{\text {ovlp }}$ results in a fast clock signal transition without any short-circuit currents. During this time the charge balancing transistor begins to close while the tristate buffers already start conducting increasing the risk of a $V D D$-to- $G N D$ short. In modern CMOS technologies this overlap time has to be reduced or eliminated in order to get good robustness against process variations.

\section{Two kinds of signal degradations and the reasons}

In the case of well adjusted charge balancing the $c b_{-} s$ pulse in Fig. 3 is as long as necessary to recycle about half of the

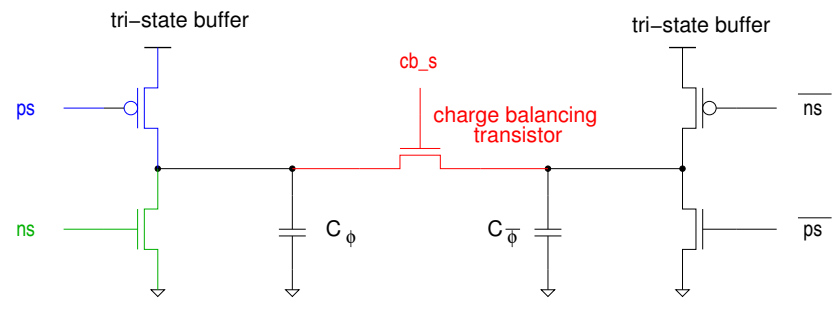

Fig. 2. Charge balancing circuit for complementary clock phases.

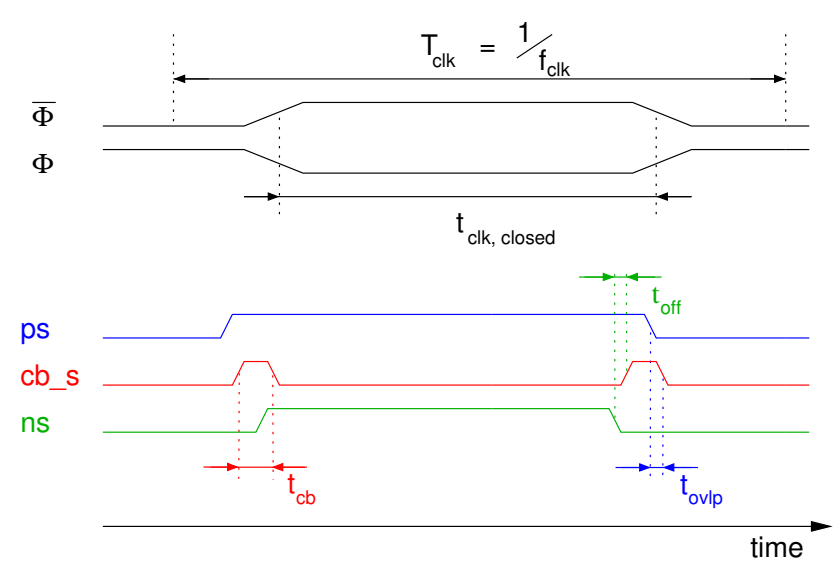

Fig. 3. Timing of control pulses and resulting clock signal phases.

charge which is stored in the clock capacitances. This leads to the best power-delay efficiency, because the clock transition time is as short as possible at the maximum recycled charge.

When the balancing time is shortened, for example due to a slower path for the event that activates the balancing transistor, the full power saving potential cannot be exploited. A clock signal waveform as illustrated in Fig. 4 will be the result. In this case the power gain towards a conventional clock system decreases. But the timing constraints for the clock transitions are fulfilled and the functionality of the logical paths in the building block is guaranteed.

An opposite situation will occur when the balancing impulse gets too long due to signal run time degradations reverse to the case explained above. The resulting waveforms are shown in Fig. 5. In this case the power gain is marginally increased. However, the functionality of the system cannot be guaranteed any longer, because clock transitions get very long which may cause timing violations for critical paths in the logic.

Timing shifts in the control signal paths are mainly caused by different propagation delay variations in the involved logic gates. The main sources of varying delays are onchip variation (OCV) of transistors, temperature drift, supply noise and process corner variations. If e.g. the system is optimized for the slow-corner, the balancing impulses in the fast-corner may degrade and in disadvantageous cases become too short to get the full voltage swing. 


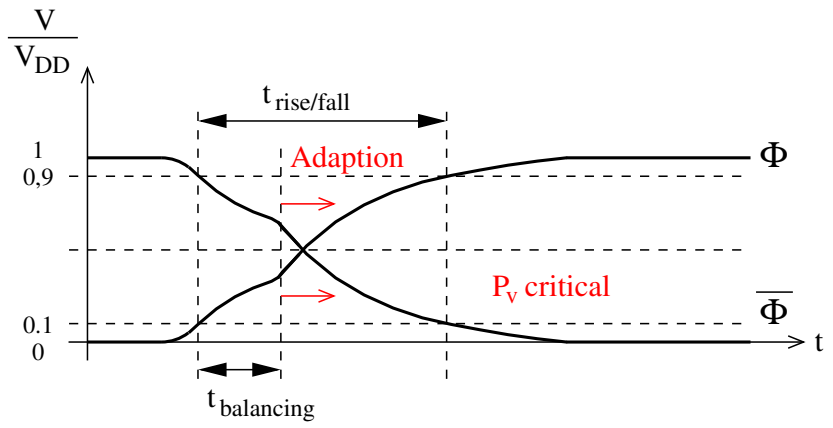

Fig. 4. Charge balancing time is too short.

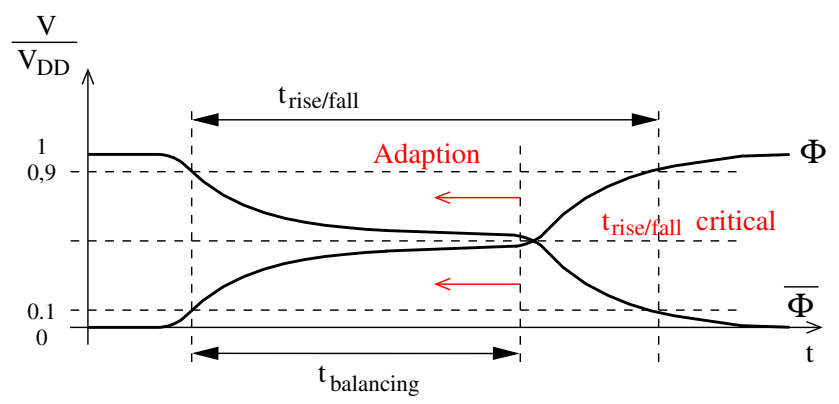

Fig. 5. Charge balancing time is too long.

\section{Adapting the charge balancing time}

To overcome the above described drawbacks we propose in this work a clock generator which is able to adapt the process of charge balancing individually for every produced chip over the relevant temperature range and in a wide interval of supply voltages. For the adaption functionality it is inevitable to fulfill some kind of measurement towards the recycling event.

As quantification of the recycled charge is complicated to realize we developed a circuit which allows an observation of the charge balancing process.

\subsection{Equilibrium detection gate}

With a simple binary information, if a voltage level near the equilibrium has been reached before the tri-state buffers are activated, an adaption decision can be made.

Figure 6 shows the new gate which is able to make a fullswing transition while both inputs, which are the complementary clock phases $\Phi$ and $\bar{\Phi}$, reach only a level of half the supply voltage - the equilibrium.

Suppose a rising transition of clock signal $\bar{\Phi}$. At the beginning input $\Phi=1$ and input $\bar{\Phi}=0$. From this it follows that both $n$-transistors on the left side conduct, because their gates are connected to $\Phi$. Both left outputs are on logical " 0 ". Accordingly the outputs on the right side are both on logical " 1 ".

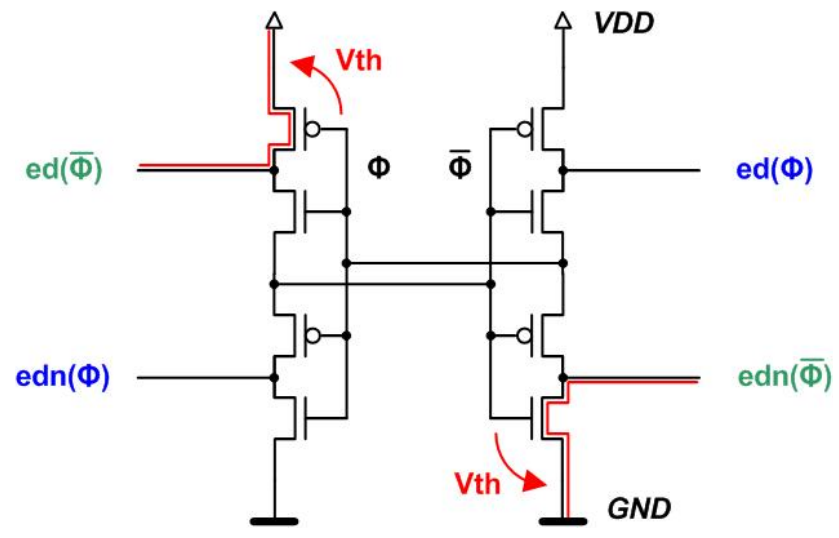

Fig. 6. Equilibrium detection gate.

When $\Phi$ is falling until $U_{\Phi}-U_{\bar{\Phi}} \approx V_{\text {th }}$ all four transistors in the middle of the gate, the devices which have no connection to $V D D$ or $G N D$ get closed. When $\Phi$ is reaching a voltage level of $U_{\Phi}=V D D-V_{\text {th }}$ the upper left p-transistor begins to conduct pulling the green equilibrium detection signal $e d(\bar{\Phi})$ to $V D D$. Analoguous the other green output $e d n(\bar{\Phi})$ on the lower right position of the gate is pulled down to GND. These synchronous switching events mark the detection point for the equilibrium.

Later on, when $U_{\Phi}-U_{\bar{\Phi}} \approx-V_{\text {th }}$ the blue output signals $e d(\Phi)$ and $e d n(\Phi)$ begin to switch. These events are not relevant for the detection of a rising edge of $\bar{\Phi}$ as explained above. $e d(\Phi)$ and $e d n(\Phi)$ can in the following half-cycle detect the equilibrium of a rising edge of $\Phi$.

\subsection{Timing relation for the equilibrium detection}

A detection of the equilibrium is not enough to make a decision if the balancing process is well adjusted or not. To get an information about the quality of the charge recycling it is important at what time the equilibrium is reached.

For this purpose a capture flip-flop is required which has to be clocked by the control signal of the according tri-state buffer. When the tri-state buffer is activated the balancing event ends and from then on charge is taken from the power supply. At this moment the voltage equilibrium should be reached if the balancing process is properly adjusted.

Therefore the capture flip-flop is closed by the tri-state activation signal (see Fig. 7). If the equilibrium detection signal comes too late it cannot enter into the capture flip-flop. The output of the flip-flop remains on logical " 0 " and gives the message: "balancing time is too short".

In this case the balancing impulse will be made a little longer by inserting more delay elements $\Delta t$ into the timing path for the charge balancing impulse and for the tri-state enable signal.

A too long balancing time is difficult to detect, so every 16 cycles the balancing impulse is shortened by one step. If this 


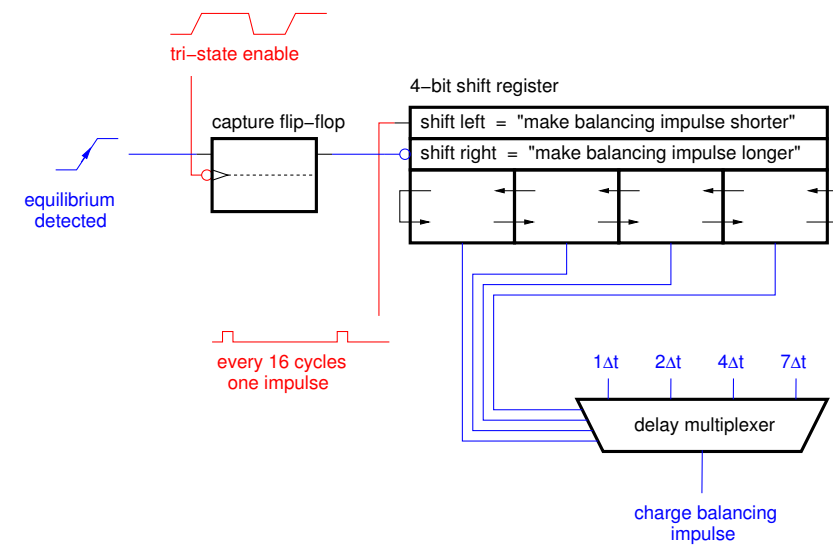

Fig. 7. Charge balancing adaption logic.

shortening was not necessary it is corrected in the following cycle by the adaption mechanism explained above.

\section{VLSI implementation of the clock generator with parameter adaption}

With the above described logic path the clock generator with parameter adaption has been implemented. For the realization in a 40-nm CMOS technology there are some further details to regard.

In order to obtain an efficient implementation a trade-off between hardware complexity and timing shift granularity has to be found. Therefore, we decided not to add the same delay increment $\Delta t$ with every step of the delay multiplexer. For a small balancing time the increment towards the next longer one is chosen small, while the increment is gradually increased with longer balancing times. With this nonequidistant delay steps an overall number of four different delays is sufficient (see Fig. 7).

For a robust functionality of the system steep transitions of the equilibrium detection signals are important. On the one hand, this can be enhanced by high threshold voltage devices for the four middle transistors in Fig. 6 which are not connected to $V D D$ or $G N D$. The higher their threshold voltage is the earlier they are switched off when a clock transition occurs and the outputs of the detection gate are earlier prepared for switching. On the other hand, the four transistors connected to $V D D$ or $G N D$ are low threshold voltage devices for fast switching in the moment the equilibrium is reached.

Figure 8 shows the layout of the 40-nm CMOS implementation of the clock generator. The adaption circuitry, positioned in the middle, accounts for about $30 \%$ of the complete area. As the clock generator usually consumes much less than $5 \%$ of the chip area of a signal processing building block, the overhead for the parameter adaption feature is almost negligible.

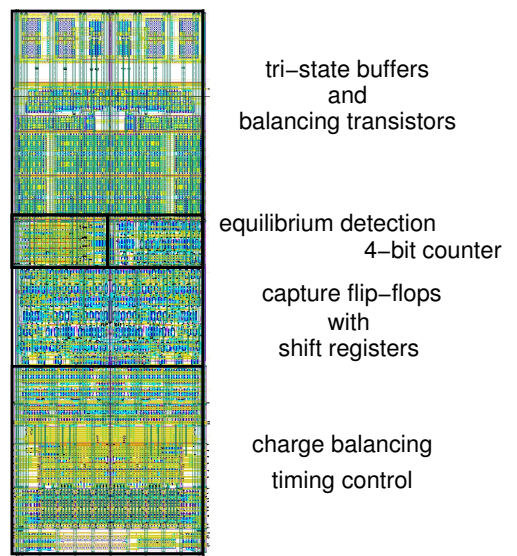

Fig. 8. Layout of the clock generator in 40-nm CMOS.

Table 1. Power dissipation @ $400 \mathrm{MHz} ; 40^{\circ} \mathrm{C} ; 650 \mathrm{mV}$.

\begin{tabular}{lccc}
\hline Corner case & $\begin{array}{c}\text { fixed } \\
\text { charge balancing }\end{array}$ & $\begin{array}{c}\text { adaptive } \\
\text { charge balancing }\end{array}$ & gain \\
\hline Slow & $558 \mu \mathrm{W}$ & $584 \mu \mathrm{W}$ & $-4.5 \%$ \\
Typical & $774 \mu \mathrm{W}$ & $627 \mu \mathrm{W}$ & $19 \%$ \\
Fast & $948 \mu \mathrm{W}$ & $714 \mu \mathrm{W}$ & $24.7 \%$ \\
\hline
\end{tabular}

\section{Benchmarking}

In this section we will compare a conventional clock generator and one with charge balancing but without parameter adaption to the proposed new clock generator with parameter adaption for the charge balancing feature.

In Table 1 the power dissipation of both charge balancing implementations is summarized for the classical process corners at a total clock capacitance of $4 \times C_{\Phi}$ with $C_{\Phi}=1 \mathrm{pF}$.

One drawback of the system without parameter adaption is, that the fixed timing for the balancing pulses has to be a compromise over all corner cases. The adaptive system switches into another delay multiplexer position for each of the three corner cases. In the slow-corner, for which the frequency constraint of the application has to be met, the conventional system is optimized and has a small advantage over the adaptive system. But in the typical- and fast-corner the power efficiency of the adaptive system is by about $20 \%$ superior.

In Fig. 9 the power dissipation of both clock systems is illustrated over the relevant temperature interval for three different capacitive loads of a clock tree at slow $/ 650 \mathrm{mV}$.

The system with fixed charge balancing, which is well adjusted for the timing worst case at a temperature of $0^{\circ} \mathrm{C}$, gets out of the recycling optimum with rising temperature. The adaptive system switches into a longer balancing time, when the circuit becomes faster with increasing temperature, which means a higher recycling gain. 


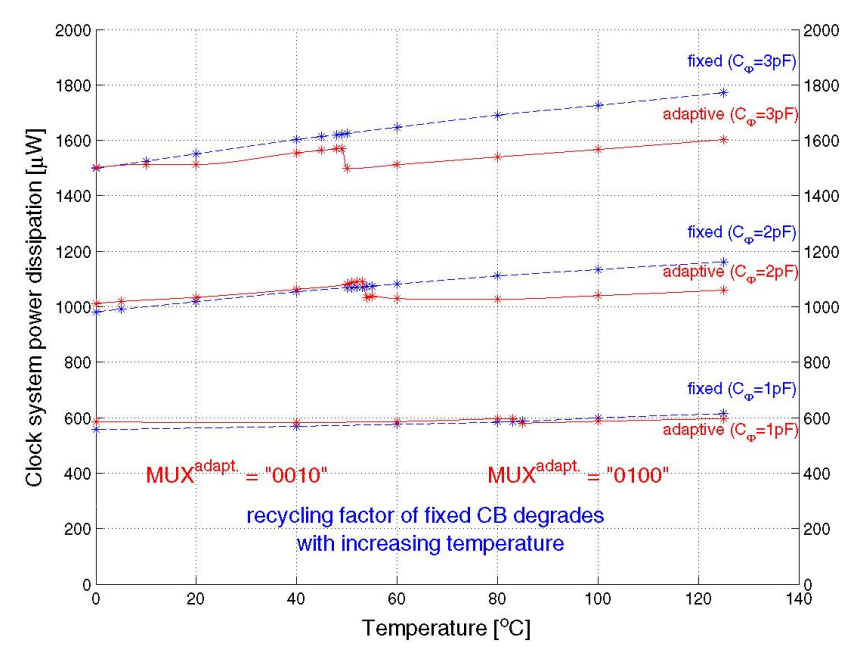

Fig. 9. Power dissipation over the relevant temperature interval.

Table 2. Clock power: Slow-corner vs. Monte Carlo simulation.

\begin{tabular}{lccc}
\hline Corner case & $\begin{array}{c}\text { no } \\
\text { charge bal. }\end{array}$ & $\begin{array}{c}\text { fixed } \\
\text { charge bal. }\end{array}$ & $\begin{array}{c}\text { adaptive } \\
\text { charge bal. }\end{array}$ \\
\hline $\begin{array}{l}\text { Slow } \\
\text { gain }\end{array}$ & $719 \mu \mathrm{W}$ & $558 \mu \mathrm{W}$ & $584 \mu \mathrm{W}$ \\
\hline $\begin{array}{l}\text { Monte Carlo } \\
\text { Global: slow }\end{array}$ & $746 \mu \mathrm{W}$ & $705 \mu \mathrm{W}$ & $659 \mu \mathrm{W}$ \\
gain & - & $5 \%$ & $11 \%$ \\
\hline
\end{tabular}

Finally, a monte-carlo benchmark is presented in Table 1 over all three clock systems mentioned above. The clock system with fixed charge balancing suffers much from device variations. A worst scenario taken from monte-carlo simulations reduces the gain towards the conventional clock system by more than three-quarters. To some extent the adaption logic is able to compensate these degradations. The resulting gain of the adaptive approach is more than twice as high as without parameter adaption.

\section{Conclusion}

In a clock system with complementary phases the power dissipation can be reduced by about $30 \%$ with the implementation of a charge balancing approach. One drawback of this approch, the compromise which has to be made for the balancing time over all process corners can be very good compensated by an adaption logic. The adaptive clock generator proposed in this work has an about $20 \%$ lower power dissipation in the typical- and fast-corner towards the same clock generator without adaption feature. This means a total power reduction within a high performance number-crunching unit of more than $10 \%$.

Verifying the clock system with monte carlo-simulation reveals, that a charge balancing approach suffers much from process variation. The gain towards a conventional clock system can be more than doubled by applying a parameter adaption to the charge balancing mechanism.

For future research an alternative adaption logic may be possible by using an equilibrium detection gate based on a short circuit measurement.

\section{References}

Das, B. P., Amrutur, B., Jamadagni, H. S., Arvind, N. V., and Visvanathan, V.: Within-Die Gate Delay Variability Measurement Using Reconfigurable Ring Oscillators, IEEE Transactions on Semiconductor Manufacturing, 22, 256-267, 2009.

Kyriakis-Bitzaros, E. D. and Nikolaidis, S.: Design of Low Power CMOS Drivers Based on Charge Recycling Technique, IEEE Symposium on Circuits and Systems, pp. 1924-1927, 1997.

Jaffari, J. and Anis, M.: Variability-Aware Device Optimization under $\mathrm{I}_{\mathrm{on}}$ and Leakage Current Constraints, International Symposium on Low Power Electronics and Design, 2006.

Pang, L.-T., Qian, K., Spanos, C. J., and Nikolic, B.: Measurement and Analysis of Variability in $45 \mathrm{~nm}$ Strained-Si CMOS Technology, IEEE Journal of Solid-State Circuits, 44, 2233-2243, 2009.

Nassif, S., Bernstein, K., Frank, D. J., Gattiker, A., Haensch, W., Ji, B. L., Nowak, E., Pearson, D., and Rohrer, N. J.: High Performance CMOS Variability in the $65 \mathrm{~nm}$ Regime and Beyond, IEEE International Electron Devices Meeting, pp. 569571, 2007.

Noll, T. G. and de Man, E.: Pushing the Performance Limits due to Power Dissipation of Future ULSI Chips, IEEE International Symposium on Circuits and Systems, 4, 1652-1655, 1992. 